\title{
QUASI-CONVEXITY AND LOWER SEMI-CONTINUITY OF MULTIPLE VARIATIONAL INTEGRALS OF ANY ORDER
}

\author{
BY \\ NORMAN G. MEYERS
}

Introduction. The purpose of this paper is the determination of necessary and sufficient conditions for the lower semi-continuity, under various kinds of convergence, of integrals of the form

$$
I(u ; \Omega)=\int_{\Omega} f\left(x, u(x), \cdots, D^{l} u(x)\right) d x .
$$

Here $x=\left(x^{1}, \cdots, x^{n}\right), u=\left(u^{1}, \cdots, u^{m}\right), \Omega$ is a bounded domain and the integrand $f\left(x, p^{0}, \cdots, p^{l}\right)$ is a continuous function of its arguments.

In 1952 Morrey studied the case $l=1$ and introduced the concept of quasiconvexity (see [3]). Extending this concept to the cases $l \geqq 1$, we say that an integrand $f\left(p^{l}\right)$ is quasi-convex if each polynomial of degree $\leqq l$ minimizes the integral, $\int_{\Omega} f\left(D^{l} u(x)\right) d x$, among all functions whose derivatives of order $l-1$ satisfy a Lipschitz condition on $\Omega$ (we denote this function space by $\mathscr{W}^{l, \infty}(\Omega)$ ) and assume the same Dirichlet data on $\partial \Omega$ as the polynomial. The reason for the term quasi-convexity becomes clear when one sees that convexity implies quasiconvexity and quasi-convexity in turn implies the Legendre condition (at least for smooth integrands) which contains within it various convexities. Hence, quasi-convexity is a condition which falls between convexity and a weaker kind of convexity.

In Theorems 1 and 2 of $\S 2$, I extend Morrey's results by showing that the necessary and sufficient condition for lower semi-continuity of $I(u ; \Omega)$ in $\mathscr{W}^{l, \infty}(\Omega)$, under uniform convergence of derivatives of order $\leqq l-1$ and uniform boundedness of derivatives of order $l$, is that $f\left(x, p^{0}, \cdots, p^{l}\right)$ be quasi-convex in $p^{l}$ for each fixed value of the variables $\left(x, p^{0}, \cdots, p^{l-1}\right)$. The proof is a straightforward extension of Morrey's for the case $l=1$. However, it contains the added feature that the necessity is derived asssuming only that the admissible functions satisfy fixed Dirichlet boundary conditions.

I then go on to consider lower semi-continuity under weak convergence in a space $\mathscr{W}^{l, r}(\Omega)(1 \leqq r<\infty)$, the space of functions with strong derivatives up to the order $l$ which are in $\mathscr{L}^{r}(\Omega)$. Two cases are considered, though they do not require separate treatment: first, the case where the admissible functions satisfy a fixed Dirichlet boundary condition, and second, the case of no boundary condition.

Received by the editors July 23, 1964. 
In addition to quasi-convexity, a new condition, too involved to state here, enters the picture. In Theorem 4 of $\S 4$, I show that quasi-convexity and this second condition are necessary and sufficient for lower semi-continuity when the integrand has certain reasonable growth properties. This second condition, though hard to get at in general, is easily seen to be satisfied in many important cases; for example it is satisfied if the integrand is bounded below by a constant. Moreover, it can usually be eliminated when $l=1$ and the admissible functions satisfy fixed Dirichlet boundary conditions (see Theorem 5 in $\S 4$ ). I have not been able to derive a comparable result for $l>1$ but believe that it is true (see the conjecture following the proof of Theorem 5).

The results of $\S 4$ are somewhat more general than Morrey's, who restricts himself to integrands bounded below and demands that the functions converge uniformly. The outline of the method of proof is Morrey's. However, I wish to emphasize that the extension of Morrey's method to the case $l>1$ is not immediate since certain serious technical problems arise. The crux of the proof is Morrey's ingenious idea of using an auxiliary strongly convergent sequence. Although the construction of the strongly convergent sequence is an elementary matter in the case $l=1$, it does not appear to be so for $l>1$. I carry out the construction in a series of lemmas contained in $\$ 3$. It is based on the results of Agmon, Douglis and Nirenberg concerning Poisson kernels for elliptic equations (see [1]). Curiously enough, the estimates given in [1] for solutions of elliptic boundary problems are not adequate, so that we cannot construct the sequence simply by taking solutions of elliptic boundary problems, whose existence is known, and applying the a priori estimates.

The paper also contains some results for integrands which grow more rapidly, given in Theorem 6 of $\S 4$, and in $\S 5$ closes with the derivation of the Legendre condition from quasi-convexity.

1. Notation and preliminary definitions. $\mathscr{R}^{n}$ will denote the real $n$-dimensional space of vectors (points) $x=\left(x^{1}, \cdots, x^{n}\right)$ with norm of $x=|x|=\left(\sum_{i=1}^{n}\left(x^{i}\right)^{2}\right)^{1 / 2}$. $u=u(x)=\left(u^{1}(x), \cdots, u^{m}(x)\right)$ will denote a function defined on some subset of $\mathscr{R}^{n}$ which assumes values in $\mathscr{R}^{m}$. For $i=1, \cdots, n$ and $j=1,2, \cdots, D_{i}^{j} u$ will be the vector-valued function $\partial^{j} u /\left(\partial x^{i}\right)^{j}$, while $D_{i}^{0} u=u$. For $\alpha$ equal to a multiindex $\left(\alpha_{1}, \cdots, \alpha_{n}\right)$ having nonnegative integral components, $D^{\alpha} u=D_{1}^{\alpha_{1}} \cdots D_{n}^{\alpha_{n}} u$. Setting $|\alpha|=\alpha_{1}+\cdots+\alpha_{n}$ and $l=0,1, \cdots, D^{l} u$ will stand for the vector-valued function whose components are all the components of the $D^{\alpha} u$ for $|\alpha|=l$ and $D^{l} u$ will stand for the vector-valued function whose components are all the components of the $D^{\alpha} u$ for $|\alpha| \leqq l$.

Let $\Omega$ be a domain (open connected set) in $\mathscr{R}^{n}$. For $l=0, \cdots, \infty$ we define $\mathscr{C}^{l}(\Omega)$ to be the linear space of functions $u(x)$ all of whose derivatives of order $\leqq l$ exist and are continuous on $\Omega . \mathscr{C}_{0}^{l}(\Omega)$ will be the subspace of $\mathscr{C}^{l}(\Omega)$ consisting of functions with compact support contained in $\Omega . \mathscr{C}^{l}(\bar{\Omega})$ will be the subspace of 
$\mathscr{C}^{l}(\Omega)$ consisting of functions whose derivatives of order $\leqq l$ have continuous extensions to $\bar{\Omega}$. Of course, the above spaces depend on the value of $m$ but this is omitted from the notation since it will be clear what is meant in any given case, and we adopt the same policy throughout the paper.

As usual, $\mathscr{L}^{r}(\Omega)(1 \leqq r<\infty)$ is the Banach space of Lebesgue measurable functions $u(x)$ defined almost everywhere in $\Omega$ for which

$$
\|u\|_{\mathscr{L}^{r}(\Omega)}=\left(\int_{\Omega}|u(x)|^{r} d x\right)^{1 / r}<\infty .
$$

For $l=0,1, \cdots$ and $1 \leqq r<\infty$, we define $\mathscr{W}^{l, r}(\Omega)$ to be the Banach space of functions $u(x)$ with strong derivatives $D^{l} u$ in $\mathscr{L}^{r}(\Omega)$ with the norm

$$
\|u\|_{\mathscr{W}^{l, r}(\Omega)}=\left\|D^{l} u\right\|_{\mathscr{E}^{r}(\Omega)} .
$$

$\mathscr{W}_{0}^{l, r}(\Omega)$ is the closure of $\mathscr{C}_{0}^{\infty}(\Omega)$ in $\mathscr{W}^{l, r}(\Omega)$.

We now extend the definition of the $\mathscr{W}$-spaces to the case $r=\infty . \mathscr{W}^{l, \infty}(\Omega)$ is the linear space of functions $u(x)$ with strong derivatives $D^{l} u$ such that $\left\|\boldsymbol{D}^{l} u\right\|_{\mathscr{L}^{\infty}(\Omega)}=\operatorname{ess}_{\Omega} \sup \left|\boldsymbol{D}^{l} u\right|<\infty$. Instead of norming this space we define convergence of sequences in $\mathscr{W}^{l, \infty}(\Omega)$, which makes it a complete linear topological space. We say

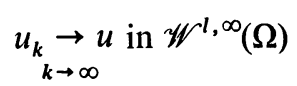

if

and

$$
\left\|D^{l-1} u_{k}-D^{l-1} u\right\|_{\mathscr{L}^{\infty}(\Omega)} \rightarrow 0 \text { as } k \rightarrow \infty
$$

$$
\left\|D^{l} u_{k}\right\|_{\mathscr{L}^{\infty}(\Omega)} \leqq M<\infty \text { for all } k .
$$

In analogy with the case of finite $r$, we take $\mathscr{W}_{0}^{l, \infty}(\Omega)$ to be the closure of $\mathscr{C}_{0}^{\infty}(\Omega)$ under convergence in $\mathscr{W}^{l, \infty}(\Omega)$.

When $\Omega$ is a bounded domain, it is clear that $\mathscr{W}^{l, \infty}(\Omega)$ consists of those functions in $\mathscr{C}^{l-1}(\bar{\Omega})$ for which $D^{l-1} u$ satisfies a Lipschitz condition on $\Omega$ and one can easily show that $\mathscr{W}_{0}^{l, \infty}(\Omega)$ consists precisely of those functions in $\mathscr{W}^{l, \infty}(\Omega)$ for which $D^{l-1} u(x)=0$ on $\partial \Omega$. Therefore, to say that $D^{l-1} u=D^{l-1} v$ on $\partial \Omega$ is the same as saying $u-v$ is in $\mathscr{W}_{0}^{l, \infty}(\Omega)$. In general we shall say that $u(x)$ and $v(x)$ in $\mathscr{W}^{l, r}(\Omega)$ assume the same Dirichlet data on $\partial \Omega$ in the sense of $\mathscr{W}^{l, r}(\Omega)$ if $u-v$ is in $\mathscr{W}_{0}^{l, r}(\Omega)$ and we shall call any translate of $\mathscr{W}_{0}^{l, r}(\Omega)$ a Dirichlet class of $\mathscr{W}^{l, r}(\Omega)$.

We shall use the notation $\stackrel{s}{\rightarrow}$ and $\stackrel{W}{\rightarrow}$ which mean "converges strongly to" and "converges weakly to" respectively.

2. Lower semi-continuity with respect to convergence in $\mathscr{W}^{l, \infty}(\Omega)$. It is clear that when $\Omega$ is a bounded domain, $\mathscr{W}^{l, \infty}(\Omega) \subset \mathscr{W}^{l, r}(\Omega)$, and because weak convergence in $\mathscr{W}^{l, r}(\Omega)(r<\infty)$ is equivalent to weak convergence of the respective derivatives in $\mathscr{L}^{r}(\Omega)$, convergence in $\mathscr{W}^{l, \infty}(\Omega)$ implies weak convergence in $\mathscr{W}^{l, r}(\Omega)$ but does not imply strong convergence. Therefore, any condition which is necessary for lower semicontinuity in $\mathscr{W}^{l, \infty}(\Omega)$ is also necessary for lower semi-continuity under 
weak convergence in $\mathscr{W}^{l, r}(\Omega)$. In this section we show that quasi-convexity of the integrand is necessary and sufficient for lower semi-continuity in $\mathscr{W}^{l, \infty}(\Omega)$. This is the first step in establishing similar conditions in the case of finite values of $r$.

Let $f=f\left(x, p^{l}\right)$ denote a real-valued integrand with the independent vector variable $\boldsymbol{p}^{l}$ corresponding to $\boldsymbol{D}^{l} u$. Similarly $p^{0}, \cdots, p^{l}$ denote variables corresponding to $D^{0} u, \cdots, D^{l} u$ respectively. If $\Omega$ is a bounded domain in $\mathscr{R}^{n}$ and $u(x)$ is a function in an appropriate space, $\mathscr{W}^{l, r}(\Omega)$, then the functional, $\int_{\Omega} f\left(x, D^{l} u(x)\right) d x$, will be denoted variously by $I=I(u)=I(u ; \Omega)$. If we wish to consider the functional $I$ only on a subset $\mathscr{D}$ of $\mathscr{W}^{l, r}(\Omega)$ we indicate its restriction to $\mathscr{D}$ by $I / \mathscr{D}$. If we wish to consider the integral only over $\Gamma$, a measurable subset of $\Omega$, we write $I(u ; \Gamma)$.

Definition 1 (QuASI-CONVEXITY). Let $f=f\left(p^{l}\right)$ be a continuous real-valued integrand defined for all values of $p^{l}$. Let $\Omega$ be an arbitrary bounded domain in $\mathscr{R}^{n}$. Set

$$
I(u ; \Omega)=\int_{\Omega} f\left(D^{l} u(x)\right) d x \text { for } u(x) \text { in } \mathscr{W}^{l, \infty}(\Omega) .
$$

We say that $f\left(p^{l}\right)$ is quasi-convex if

$$
I(w+z ; \Omega) \geqq I(w ; \Omega)
$$

for every polynomial $w(x)$ of degree $\leqq l$, every function $z(x)$ in $\mathscr{W}_{0}^{l, \infty}(\Omega)$ and every $\Omega$.

Inequality (2) states that $I(u ; \Omega)$ assumes an absolute minimum, among all $u(x)$ in the Dirichlet class of $w(x)$, at $u=w$. Since $D^{l} w$ is an arbitrary constant vector $c$, the inequality (2) can be rewritten in the form

$$
\int_{\Omega} f\left(c+D^{l} z(x)\right) d x \geqq f(c) \cdot \text { meas } \Omega \text {. }
$$

In verifying quasi-convexity it is sufficient to fix the domain $\Omega$. For let us suppose that (2) or equivalently (3) has been verified for $\Omega$ and let $\Omega^{\prime}$ be some other bounded domain. Then for the proper choice of the scalar constant $a \neq 0$ and the point $x_{0}$, the transformation $x=a x^{\prime}+x_{0}$ maps $\Omega^{\prime}$ onto $\Omega^{\prime \prime}$, a subdomain of $\Omega$, and

$$
\int_{\Omega^{\prime}} f\left(c+D_{x^{\prime}}^{l} z\left(x^{\prime}\right)\right) d x^{\prime}=|a|^{-n} \int_{\Omega^{\prime \prime}} f\left(c+D_{x^{\prime}}^{l} z\left(\frac{x-x_{0}}{a}\right) \cdot a^{l}\right) d x .
$$

Since $z\left(\left(x-x_{0}\right) / a\right)$ can be considered to vanish identically outside of $\Omega^{\prime \prime}$ and as such is in $\mathscr{W}_{0}^{l, \infty}(\Omega)$, the second term in (4)

$$
=|a|^{-n} \int_{\Omega} f\left(c+D_{x}^{l} z\left(\frac{x-x_{0}}{a}\right) \cdot a^{l}\right) d x-f(c)|a|^{-n} \operatorname{meas}\left(\Omega-\Omega^{\prime \prime}\right) .\left({ }^{1}\right)
$$

(1) Here we use the fact that $D_{x}^{l} z\left(\left(x-x_{0}\right) / a\right)=0$ a.e. on $\partial \Omega^{\prime \prime}$ which might have positive measure. In general it can be shown that strong derivatives vanish a.e. on a set where a function is constant. 


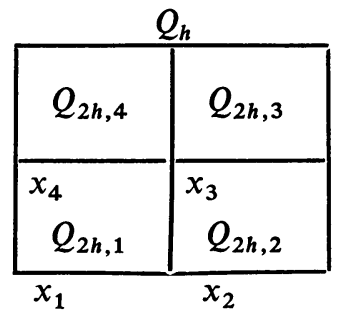

Figure 1. (The case $n=2, k=2$.)

But this is

$$
\geqq f(c)|a|^{-n} \text { meas } \Omega^{\prime \prime}=f(c) \text { meas } \Omega^{\prime} \text {. }
$$

Secondly, it is sufficient to verify (2) or (3) only for $z(x)$ in $\mathscr{C}_{0}^{\infty}(\Omega)$. For if $z(x)$ is in $\mathscr{W}_{0}^{l, \infty}(\Omega)$ it is easy to show that there exists a sequence $z_{k}(x)$ in $\mathscr{C}_{0}^{\infty}(\Omega)$ such that

and

$$
\left|D^{l} z_{k}(x)\right| \leqq M<\infty
$$

$$
D^{l} z_{k}(x) \stackrel{\text { a.e. }}{\longrightarrow} D^{l} z(x) .
$$

Therefore, if (3) holds for $z_{k}(x)$, it must also hold for $z(x)$ by the Lebesgue bounded convergence theorem.

THEOREM 1 (NECESSITY OF QUASI-CONVEXITY). Let $f=f\left(x, p^{l}\right)$ be a continuous integrand defined for all $x$ in a bounded domain $\Omega$ and all values of $p^{\iota}$, which is furthermore bounded on bounded sets of $\left(x, p^{l}\right)$-space. Define

$$
I(u ; \Omega)=\int_{\Omega} f\left(x, D^{l} u(x)\right) d x \text { for } u(x) \text { in } \mathscr{W}^{l, \infty}(\Omega) .
$$

If $\mathscr{D}$ is any Dirichlet class in $\mathscr{W}^{l, \infty}(\Omega)$ and $I / \mathscr{D}$ is lower semi-continuous relative to convergence in $\mathscr{W}^{l, \infty}(\Omega)$ then $f\left(x, p^{l-1}, p^{l}\right)$ is quasi-convex in $p^{l}$ for each fixed value of $\left(x, p^{l-1}\right)$.

Proof. Let $x_{1}$ be an arbitrary point in $\Omega$ and let $Q_{h}$ be the cube $x_{1}^{i} \leqq x^{i} \leqq x_{1}^{i}+1 / h$ $(i=1, \cdots, n)$. Let $z=z(x)$ be an arbitrary function in $\mathscr{C}_{0}^{\infty}$ (int $Q_{1}$ ) and extend $z(x)$ to all of $\mathscr{R}^{n}$ as a $\mathscr{C}^{\infty}$-function with period equal to 1 in each of the $x^{i}$. Then define

$$
\begin{aligned}
z_{h, k}(x) & =(h k)^{-l} z\left(h k\left(x-x_{1}\right)+x_{1}\right) \text { for } x \text { in } Q_{h}, \\
& =0 \text { for } x \text { not in } Q_{h}
\end{aligned}
$$

where $h, k=1,2, \cdots . z_{h, k}(x)$ is in $\mathscr{C}_{0}^{\infty}$ (int $Q_{h}$ ) and is periodic in $Q_{h}$ with $Q_{h k}$ as a period cube. Number the period cubes in $Q_{h}$ in some manner and denote them by $Q_{h k, j}\left(j=1, \cdots, k^{n}\right)$ with $Q_{h k, 1}=Q_{h k}$. Denote the corner of $Q_{h k, j}$ nearest to $x_{1}$ by $x_{j}$ (see Figure 1).

We now let $u(x)$ be a function in $\mathscr{D}$ which is a $\mathscr{C}^{\infty}$-function in some neighborhood of $x_{1}$ and for which $\left(D^{l-1} u(x), D^{l} u\left(x_{1}\right)\right)=\left(c^{l-1}, c^{l}\right), c^{l-1}$ and $c^{l}$ being arbitrarily 
chosen constant vectors. For sufficiently large values of $h, Q_{h} \subset \Omega$, so that $I\left(u+z_{h, k} ; Q_{h}\right)$ is defined and

$$
\begin{aligned}
I\left(u+z_{h, k} ; Q_{h}\right)= & \sum_{j=1}^{k^{n}} \int_{Q_{h k, j}}\left\{f\left(x, D^{l} u(x)+D^{l} z_{h, k}(x)\right)\right. \\
& \left.-f\left(x_{j}, D^{l-1} u\left(x_{j}\right), D^{l} u\left(x_{j}\right)+D^{l} z_{h, k}(x)\right)\right\} d x \\
& +\sum_{j=1}^{k^{n}} \int_{Q_{h k}, j} f\left(x_{j}, D^{l-1} u\left(x_{j}\right), D^{l} u\left(x_{j}\right)+D^{l} z_{h, k}(x)\right) d x .
\end{aligned}
$$

It is clear that $z_{h, k}(x) \rightarrow 0$ in $\mathscr{W}^{l, \infty}(\Omega)$ as $k \rightarrow \infty$. Further, if $h$ is sufficiently large, $u(x)$ is in $\mathscr{C}^{\infty}\left(Q_{h}\right)$ and it follows by an obvious argument, using the continuity of $f$ and $D^{l} u$ and the uniform convergence to zero of the $D^{l-1} z_{h, k}$, that the first sum in (6) tends to 0 as $k \rightarrow \infty$.

To handle the second sum in (6), perform the change of variables $y=h k\left(x-x_{j}\right)$ $+x_{1}$ in the $j$ th integral. This maps the cube $Q_{h k, j}$ onto the cube $Q_{1}$ in each case and recalling the definition of the $z_{h, k}(x)$ in (5) and the periodicity of $z(x)$, the second sum is seen to reduce to

$$
\sum_{j=1}^{k^{n}}(h k)^{-n} \int_{Q_{1}} f\left(x_{j}, D^{l-1} u\left(x_{j}\right), D^{l} u\left(x_{j}\right)+D^{l} z(y)\right) d y .
$$

(7) being a Riemann sum, tends, as $k \rightarrow \infty$, to

(8) $\quad \lim _{k \rightarrow \infty} I\left(u+z_{h, k} ; Q_{h}\right)=\int_{Q_{h}} \int_{Q_{1} l^{l}} f\left(x, D^{l-1} u(x), D^{l} u(x)+D^{l} z(y)\right) d y d x$.

Since we are assuming the lower semi-continuity of $I / \mathscr{D}$, it follows that

$$
\begin{aligned}
\lim _{k \rightarrow \infty} I\left(u+z_{h, k} ; \Omega\right) & =\lim _{k \rightarrow \infty} I\left(u+z_{h, k} ; Q_{h}\right)+I\left(u ; \Omega-Q_{h}\right) \\
& \geqq I\left(u ; Q_{h}\right)+I\left(u ; \Omega-Q_{h}\right) .
\end{aligned}
$$

Hence from (8)

$$
\int_{Q_{h}} \int_{Q_{1}} f\left(x, D^{l-1} u(x), D^{l} u(x)+D^{l} z(y)\right) d y d x \geqq \int_{Q_{h}} f\left(x, D^{l} u(x)\right) d x .
$$

Multiplying both sides of (9) by $h^{n}$ and letting $h \rightarrow \infty$ yields

$$
\int_{Q_{1}} f\left(x_{1}, c^{l-1}, c^{l}+D^{l} z(y)\right) d y \geqq f\left(x_{1}, c^{l-1}, c^{l}\right),
$$

from which we infer the quasi-convexity.

LEMMA 1. Let $I$ be defined as in Theorem 1 and let $\mathscr{D}$ be a Dirichlet class in $\mathscr{W}^{l, \infty}(\Omega)$. If $I / \mathscr{D}$ achieves an absolute minimum at $w(x)$, then $I$ is lower semicontinuous at $w(x)$. 
Proof. Let $z_{k}(x) \rightarrow 0$ in $\mathscr{W}^{l, \infty}(\Omega)$ as $k \rightarrow \infty$. Let $\phi(x)$ be in $\mathscr{C}_{0}^{\infty}(\Omega)$ with $0 \leqq \phi(x) \leqq 1$. Then

$$
\begin{aligned}
I\left(w+z_{k}\right)= & I\left(w+z_{k}\right)-I\left(w+\phi z_{k}\right)+I\left(w+\phi z_{k}\right) \\
= & I\left(w+z_{k} ; \Gamma\right)-I\left(w+\phi z_{k} ; \Gamma\right) \\
& +I\left(w+z_{k} ; \Omega-\Gamma\right)-I\left(w+\phi z_{k} ; \Omega-\Gamma\right) \\
& +I\left(w+\phi z_{k}\right) \\
= & J_{1}+J_{2}+J_{3} .
\end{aligned}
$$

In (11), $\Gamma$ is the largest open subset of $\Omega$ on which $\phi(x) \equiv 1$. Hence $J_{1}=0$. Since $D^{l-1} z_{k} \rightarrow 0$ uniformly and the $D^{l} z_{k}$ are uniformly bounded independent of $k$, it follows easily that

$$
\limsup _{k \rightarrow \infty} J_{2} \leqq C \text { meas }(\Omega-\Gamma),
$$

where $C$ is a constant independent of the choice of $\phi(x)$. Finally by hypothesis

$$
J_{3} \geqq I(w) \text {. }
$$

Therefore if $\varepsilon>0$, by the proper choice of $\phi(x)$, it follows that

$$
\lim _{k \rightarrow \infty} \inf I\left(w+z_{k}\right) \geqq I(w)-\varepsilon
$$

and the required result is proved.

THEOREM 2 (SufFICIENCY OF QUASI-CONVEXITY). If $f=f\left(x, p^{l}\right)$ as defined in Theorem 1, is quasi-convex in $p^{l}$ for each fixed value of $\left(x, p^{l-1}\right)$ then $I(u)$ is lower semi-continuous relative to convergence in $\mathscr{W}^{l, \infty}(\Omega)$.

Proof. Let $G_{v}(v=1,2, \cdots)$ be the cubic lattice containing the cube $0 \leqq x^{i} \leqq 2^{-v}$ and let $\Gamma_{v}$ be the union of those cubes in $G_{v}$ which are contained in $\Omega$. Now let $u(x), z_{k}(x)(k=1,2, \cdots)$ be functions in $\mathscr{W}^{l, \infty}(\Omega)$ and let $z_{k}(x) \rightarrow 0$ in $\mathscr{W}^{l, \infty}(\Omega)$. If $\varepsilon>0$ then for some sufficiently large value of $v=v^{\prime}$, which depends only on $\varepsilon$,

$$
\left|I\left(u+z_{k} ; \Omega-\Gamma_{v^{\prime}}\right)\right|,\left|I\left(u ; \Omega-\Gamma_{v^{\prime}}\right)\right|<\varepsilon
$$

for all values of $k$. If $v>v^{\prime}$ then $\Gamma_{v^{\prime}}=\bigcup_{h=1}^{N_{v}} Q_{h}$ where the $Q_{h}$ are the cubes of $G_{v}$ which are contained in $\Gamma_{v^{\prime}}$. Now

$$
\begin{aligned}
I(u & \left.+z_{k} ; \Gamma_{v^{\prime}}\right) \\
& =\int_{\Gamma_{v^{\prime}}}\left\{f\left(x, D^{l} u(x)+D^{l} z_{k}(x)\right)-f\left(x, D^{l-1} u(x), D^{l} u(x)+D^{l} z_{k}(x)\right)\right\} d x \\
& +\int_{\Gamma_{v^{\prime}}} f\left(x, D^{l-1} u(x), D^{l} u(x)+D^{l} z_{k}(x)\right) d x .
\end{aligned}
$$

Obviously the first integral in (13) tends to 0 as $k \rightarrow \infty$. We rewrite the second integral in the form 


$$
\begin{aligned}
\int_{\Gamma_{. .}}\left\{f\left(x, D^{l-1} u(x), D^{l} u(x)+D^{l} z_{k}(x)\right)-f\left(\bar{x}, \overline{D^{l-1}} u(x), \overline{D^{l} u(x)}+D^{l} z_{k}(x)\right)\right\} d x \\
\quad+\sum_{h=1}^{N v} \int_{Q_{h}}\left\{f\left(\bar{x}, \overline{D^{l-1} u}(x), \overline{D^{l} u(x)}+D^{l} z_{k}(x)\right)-f\left(\bar{x}, \overline{D^{l} u}(x)\right)\right\} d x \\
\quad+\int_{\Gamma_{v^{\prime}}} f\left(\bar{x}, \overline{D^{l} u}(x)\right) d x \\
=J_{1}+J_{2}+J_{3} .
\end{aligned}
$$

Referring to (14), if $v(x)$ is an $\mathscr{L}^{1}$-function then $\bar{v}(x)$ is a new function (depending on the value of $v$ ) defined by

$$
\bar{v}(x)=\left(\text { meas } Q_{h}\right)^{-1} \int_{Q_{h}} v(y) d y \text { for } x \text { in int } Q_{h} .
$$

It is well known that $\bar{v}(x) \rightarrow v(x)$ a.e. as $v \rightarrow \infty$, so it is a simple consequence of the continuity of $f$, the boundedness of $D^{l} u$, the uniform boundedness of the $D^{l} z_{k}$ and the Lebesgue bounded convergence theorem that

$$
J_{1} \rightarrow 0 \text { and } J_{3} \rightarrow I\left(u ; \Gamma_{v^{\prime}}\right) \text { as } v \rightarrow \infty,
$$

the convergence in the first case being uniform in $k$. Since we are assuming quasiconvexity it follows immediately from Lemma 1 that for each value of $v \geqq v^{\prime}$

$$
\liminf _{k \rightarrow \infty} J_{2} \geqq 0 .
$$

From (15) and (16) one concludes that $\lim _{\inf } f_{k \rightarrow \infty} I\left(u+z_{k} ; \Gamma_{v^{\prime}}\right) \geqq I\left(u ; \Gamma_{v^{\prime}}\right)$ and thus from (12),

$$
\liminf _{k \rightarrow \infty} I\left(u+z_{k} ; \Omega\right) \geqq I(u ; \Omega)-2 \varepsilon,
$$

from which the lower semi-continuity follows.

3. Construction of appropriate functions with assigned Dirichlet data. Before discussing lower semi-continuity in the spaces $\mathscr{W}^{l, r}(\Omega)$ for finite values of $r$, we give a set of preliminary lemmas culminating in Lemma 4 , which is of crucial importance to the following work. It leads directly to Lemma 5 of $\S 4$, which plays the same role in the case of finite $r$ as Lemma 1 in the case of infinite $r$.

The problem to be dealt with is essentially the following: to find a transformation $\Im$ which maps Dirichlet data $\Phi(x)=\left\{\phi_{0}(x), \cdots, \phi_{l-1}(x)\right\}$ given on $\partial \Omega$ into functions $\mathfrak{S}(\Phi)$ defined on $\Omega$ such that $\mathfrak{S}(\Phi)$ assumes the data $\Phi$ on $\partial \Omega$ and such that if $\Phi_{k}$ is a sequence bounded in $\mathscr{W}^{l, r}(\partial \Omega) \times \cdots \times \mathscr{W}^{1, r}(\partial \Omega)$ and strongly convergent to zero in $\mathscr{W}^{l-1, r}(\partial \Omega) \times \cdots \times \mathscr{W}^{0, r}(\partial \Omega)$ (these spaces will be defined in this section) then $\mathfrak{S}\left(\Phi_{k}\right)$ is strongly convergent to zero in $\mathscr{W}^{l, r}(\Omega)$. The particular construction I give is based on the Poisson kernels for elliptic boundary problems introduced by Agmon, Douglis and Nirenberg [1]. However, since we do not care if the constructed function $\Im(\Phi)$ satisfies a differential equation, it is 
easy to see there is wide latitude in the choice of possible kernels. It is not clear that the constructed functions can be chosen to satisfy an elliptic equation since the estimates given in [1] are not adequate.

In order to avoid technical questions concerning traces of discontinuous functions on lower dimensional manifolds, which are not necessary for the purposes of this paper, we deal with $\mathscr{C}^{\infty}$-functions and domains with $\mathscr{C}^{\infty}$-boundaries, except when it is no more difficult to give the proof in a wider context. The interested reader can easily generalize these results.

For simplicity of notation we now work in the space $\mathscr{R}^{n+1}(n \geqq 1)$ whose points we designate by $(x, t)$ where $x=\left(x^{1}, \cdots, x^{n}\right) . \mathscr{R}_{+}^{n+1}$ denotes the upper half-space $t>0$. Now consider a single elliptic equation, let us say, $\Delta^{l} u=0$, in the half-space $\mathscr{R}_{+}^{n+1}$ together with Dirichlet boundary conditions given on $t=0$. Let $K_{j}=K_{j}(x, t)$ $(j=0, \cdots, l-1)$ be the set of Poisson kernels for this problem given in $\S 2$ of [1]. (In their notation the kernels are numbered from 1 to $m$.) Let $K_{j, q}=K_{j, q}(x, t)$, where $n$ and $q$ have the same parity, be auxiliary kernels as defined in $\$ 2$ of [1]. The relevant properties of these kernels follow.

Lemma 2. (i) The kernels $K_{j, q}(x, t)$ are $\mathscr{C}^{\infty}$-functions in $\overline{\mathscr{R}_{+}^{n+1}}$ except at $x=t=0$.

$$
\Delta_{x}^{(n+q) / 2} K_{j, q}(x, t)=K_{j}(x, t)
$$

except possibly at $x=t=0$.

$$
\left|D^{m} K_{j, q}(x, t)\right| \leqq C\left(|x|^{2}+t^{2}\right)^{(j+q-m) / 2}\left(1+\left|\log \left(|x|^{2}+t^{2}\right)\right|\right)
$$

and if $m \geqq j+q+1$ then the logarithmic term in the above inequality can be omitted. The constant $C$ depends only on $m$ and $q$.

(iv) If $\phi_{j}=\phi_{j}(x)$ is in $\mathscr{C}_{0}^{\infty}\left(\mathscr{R}^{n}\right)$ then

$$
u_{j}(x, t)=\int_{R} K_{j}(x-y, t) \phi_{j}(y) d y=K_{j} * \phi_{j}
$$

is in $\mathscr{C}^{\infty}\left(\mathscr{R}_{+}^{n+1}\right)$ and $D_{t}^{i} u_{j}(x, 0)=\delta_{i j} \phi_{j}(x)$ for all $i=0, \cdots, l-1$.

$u_{j}(x, t)$ also solves the differential equation but we do not care about that here. The proofs of (i)-(iv) can be found in $\$ 2$ of [1] and are of an elementary character, unlike the proofs of the deeper properties of these kernels which rely on the Calderón-Zygmund inequality. These deeper properties are unnecessary: for our purposes.

In Lemma 3 we carry out our construction in a half-space. The rest then follows by more or less standard techniques.

LEMMA 3 (ConstruCtion IN A HALF-SPACE). If $\Phi(x)=\left\{\phi_{0}(x), \cdots, \phi_{l-1}(x)\right\}$ is a set of functions in $\mathscr{W}^{l, r}\left(\mathscr{R}^{n}\right) \times \cdots \times \mathscr{W}^{1, r}\left(\mathscr{R}^{n}\right)(1 \leqq r<\infty)$ having compact support in $\mathscr{R}^{n}$ define 


$$
\mathfrak{R}(\Phi)=\sum_{j=0}^{l-1} K_{j} * \phi_{j}
$$

Let $\Omega^{\prime}$ and $\Omega$ be bounded subdomains of $\mathscr{R}^{n}$ and $\mathscr{R}_{+}^{n+1}$ respectively. Then

(i) $\Omega$ is a linear transformation of $\mathscr{C}_{0}^{\infty}\left(\mathscr{R}^{n}\right)$ into $\mathscr{C}^{\infty}\left(\overline{\mathscr{R}_{+}^{n+1}}\right)$ and $\mathfrak{R}(\Phi)$ assumes the Dirichlet data $\Phi(x)$ on $t=0$.

(ii) $\Omega$ is a linear transformation of $\mathscr{W}_{0}^{l, r}\left(\Omega^{\prime}\right) \times \cdots \times \mathscr{W}_{0}^{1, r}\left(\Omega^{\prime}\right)$ into $\mathscr{C}^{\infty}\left(\mathscr{R}_{+}^{n+1}\right)$ and a compact linear transformation of $\mathscr{W}_{0}^{l, r}\left(\Omega^{\prime}\right) \times \cdots \times \mathscr{W}_{0}^{1, r}\left(\Omega^{\prime}\right)$ into $\mathscr{W}^{l, r}(\Omega)$.

(iii) There is a function $C(\varepsilon)$ defined for all $\varepsilon>0$ and depending only on $r, \Omega^{\prime}$ and $\Omega$ such that

$$
\|\mathcal{R}(\Phi)\|_{W^{l, r}(\Omega)} \leqq \sum_{j=0}^{l-1}\left\{\varepsilon\left\|\phi_{j}\right\|_{W_{0}^{l-j, r}\left(\Omega^{\prime}\right)}+C(\varepsilon)\left\|\phi_{j}\right\|_{W_{0}^{l-j-1, r\left(\Omega^{\prime}\right)}}\right\}
$$

for all $\Phi(x)$ in $\mathscr{W}_{0}^{l, r}\left(\Omega^{\prime}\right) \times \cdots \times \mathscr{W}_{0}^{1, r}\left(\Omega^{\prime}\right)$.

Proof. Statement (i) and the first part of (ii) follow immediately from (i) and (iv) of Lemma 2. We now turn to the second part of (ii). We compute the derivatives of $\mathcal{R}(\Phi)$ at points in $\mathscr{R}_{+}^{n+1}$ by considering the derivatives $D^{m} K_{j} * \phi_{j}, 0 \leqq m \leqq l$. Three cases are considered.

Case 1. $m-j<0$. We then have

$$
D^{m}\left(K_{j} * \phi_{j}\right)=\left(D^{m} K_{j}\right) * \phi_{j}
$$

Case 2. $m-j \geqq 0$ and even. For suitably large values of $q$ we can write

$$
\begin{aligned}
D^{m}\left\{K_{j} * \phi_{j}\right\} & =\left(D^{m} \Delta_{x}^{(n+q+j-m) / 2} \Delta_{x}^{(m-j) / 2} K_{j, q}\right) * \phi_{j} \\
& =\left(D^{m} \Delta_{x}^{(n+q+j-m) / 2} K_{j, q}\right) * \Delta_{x}^{(m-j) / 2} \phi_{j}
\end{aligned}
$$

Case 3. $m-j>0$ and odd. For suitably large values of $q$ we can write

$$
\begin{aligned}
D^{m}\left\{K_{j} * \phi_{j}\right\} & =\left(D^{m} \Delta_{x}^{(n+q+j-m-1) / 2} \Delta_{x}^{(m-j+1) / 2} K_{j, q}\right) * \phi_{j} \\
& =\left(\sum_{i=1}^{n} D^{m} D_{x^{i}} \Delta_{x}^{(n+q+j-m-1) / 2} D_{x^{i}} \Delta_{x}^{(m-j-1) / 2} K_{j, q}\right) * \phi_{j} \\
& =\sum_{i=1}^{n}\left\{\left(D^{m} D_{x^{i}} \Delta_{x}^{(n+q+j-m-1) / 2} K_{j, q}\right) * D_{x^{i}} \Delta_{x}^{(m-j-1) / 2} \phi_{j}\right\}
\end{aligned}
$$

Letting $K(x, t)$ denote the kernels in the resultant convolutions in Cases 1-3, we see that in Case 1

$$
|K(x, t)| \leqq C\left(|x|^{2}-t^{2}\right)^{(j-m-n) / 2}\left(1+\left|\log \left(|x|^{2}+t^{2}\right)\right|\right),
$$

while in Cases 2 and 3

$$
|K(x, t)| \leqq C\left(|x|^{2}+t^{2}\right)^{-n / 2},
$$

So in any case we may say that on each bounded subset of $\mathscr{R}_{+}^{n+1}, K(x, t)$ satisfies 


$$
|K(x, t)| \leqq C\left(|x|^{2}+t^{2}\right)^{-n / 2}
$$

where $C$ depends on the subset under consideration.

Before proceeding further we here adopt the convention that $\mathscr{L}^{r}\left(R^{\prime}\right)$, where $R^{\prime}$ is a positive number, stands for the space $\mathscr{L}^{r}$ over the domain $\left\{|x|<R^{\prime}\right.$, $\left.0<t<R^{\prime}\right\}$ if the domain is in $\mathscr{R}_{+}^{n+1}$ and stands for the space $\mathscr{L}^{r}$ over $\left\{|x|<\mathscr{R}^{\prime}\right\}$ if the domain is in $\mathscr{R}^{n}$. The proper interpretation will be clear from the context. Also we introduce the norm

$$
\|K\|_{\mathscr{L}^{1}\left(R^{\prime}\right) ; \mathscr{L}^{r}\left(R^{\prime \prime}\right)}=\left(\int_{0}^{R^{\prime \prime}}\left(\int_{|x|<R^{\prime}}|K(x, t)| d x\right)^{r} d t\right)^{1 / r} .
$$

Now consider a convolution of the form

$$
K * \psi=v(x, t)
$$

where $K(x, t)$ is a $\mathscr{C}^{\infty}$-function in $\overline{\mathscr{R}_{+}^{n+1}}$ except at $x=t=0$ and satisfies the condition set forth in (22). $\psi=\psi(x)$ is assumed to be a function in $\mathscr{L}^{\text {r }}\left(\mathscr{R}^{n}\right)$ with supp $\psi \subset\{|x|<R\}$. Using inequality (22) and setting $y=x / t$ we get

$$
\int_{|x|<2 R}|K(x, t)| d x \leqq C \int_{|y| \leqq 2 R / t}\left(1+|y|^{2}\right)^{-n / 2} d y .
$$

Restricting $t$ to the interval $0<t<R$ we see that the right hand integral is of the order $\log (2 R / t)$. Thus

$$
\int_{|x|<2 R}|K(x, t)| d x \leqq C \log \left(\frac{2 R}{t}\right) \quad(0<t<R)
$$

where $C$ depends only on $R$. Therefore we conclude that $\|K \mid\| \|_{\mathscr{L}^{1}(2 R) ; \mathscr{L r}_{(R)}}$ is finite. An elementary calculation using the Hölder inequality now shows

$$
\|v\|_{\mathscr{L}^{r}(R)} \leqq\|K\|_{\mathscr{L}^{1}(2 R) ; \mathscr{L} r(R)}\|\psi\|_{\mathscr{L}^{r}(R)} \text {, }
$$

so that

$$
\|v\|_{\mathscr{L}^{r}(R)} \leqq C\|\psi\|_{\mathscr{L} r(R)}
$$

where $C$ depends only on $R$.

Let $(\xi, \tau)$ be an arbitrary point of the cylinder $\{|\xi|<1 ; \tau \geqq 0\}$. Then by the same calculation that gave $(25)$ we get

$$
\begin{aligned}
& \|v(x+\xi, t+\tau)-v(x, t)\|_{\mathscr{L}^{r}(R)} \\
& \quad \leqq\|K(x+\xi, t+\tau)-K(x, t)\|_{\mathscr{L}^{1}(2 R+1) ; \mathscr{L r}^{r}(R)} \cdot\|\psi\|_{\mathscr{L} r^{r}(R)}
\end{aligned}
$$

By breaking the $t$-integration into two parts, one over a small interval $0<t \leqq R^{\prime}$ and the second over $R^{\prime}<t<R$, one sees from (24) with $2 R$ replaced by $2 R+1$ and from the continuity of $K$, that 


$$
\|K(x+\xi, t+\tau)-K(x, t)\|_{\mathscr{L}^{1}(2 R+1) ; \mathscr{L}(R)} \rightarrow 0 \text { as }(\xi, \tau) \rightarrow 0
$$

for each fixed $R$. Therefore there is a modulus of continuity $\mu$, depending on $R$ such that

$$
\|v(x+\xi, t+\tau)-v(x, t)\|_{\mathscr{L} r(R)} \leqq \mu(|\xi|+\tau) \cdot\|\psi\|_{\mathscr{L} r(R)}
$$

for $\tau \geqq 0$ and $|\xi|<1$. Therefore from (25) and (27) we see that the transformation $\psi \rightarrow K * \psi$ maps every set of functions which is bounded in $\mathscr{L}^{r}\left(\mathscr{R}^{n}\right)$ and whose supports lie in a fixed compact subset of $\mathscr{R}^{n}$, into a compact subset of $\mathscr{L}^{r}(R)$ for every $R$. Returning now to the original situation it follows that the transformation $\Omega$ must map bounded subsets of $\mathscr{W}_{0}^{l, r}\left(\Omega^{\prime}\right) \times \cdots \times \mathscr{W}_{0}^{1, r}\left(\Omega^{\prime}\right)$ into compact subsets of $\mathscr{W}^{l, r}(\Omega)$. This completes the proof of (ii).

Before proving statement (iii) let us note a further property of the transformation $\mathfrak{K}$. It is an obvious consequence of (iii) but we need it to prove (iii).

$$
\begin{gathered}
\text { If a sequence } \Phi_{k}(x) \text { converges strongly to zero in } \\
\mathscr{W}_{0}^{l-1, r}\left(\Omega^{\prime}\right) \times \cdots \times \mathscr{W}_{0}^{0, r}\left(\Omega^{\prime}\right) \text { and is bounded in } \mathscr{W}_{0}^{l, r}\left(\Omega^{\prime}\right) \times \cdots \\
\times \mathscr{W}_{0}^{1, r}\left(\Omega^{\prime}\right) \text { then } \Omega\left(\Phi_{k}\right) \text { converges to zero strongly in } \mathscr{W}^{l, r}(\Omega)
\end{gathered}
$$

The proof is simple. Using formulas (17)-(19) and (26) it follows that $\mathcal{R}\left(\Phi_{k}\right)$ converges to zero strongly in $\mathscr{W}^{l-1, r}(\Omega)$. Since $\Omega$ is compact $\Omega\left(\Phi_{k}\right)$ has a limit point in $\mathscr{W}^{l, r}(\Omega)$. But then the only possible limit point can be zero, thus proving (28).

Let us now suppose that (iii) is false. In that case there must exist an $\varepsilon>0$ and a sequence $\Phi_{k}(x)$ such that

$$
\sum_{j=0}^{l-1}\left\|\phi_{j, k}\right\|_{\mathscr{W}_{0}^{l-j, r}\left(\Omega^{\prime}\right)}=1 \text { for all } k
$$

and

$$
\lim _{k \rightarrow \infty} \frac{\left\|\boldsymbol{\Omega}\left(\Phi_{k}\right)\right\|_{W^{l, r}(\Omega)}-\varepsilon}{\sum_{j=0}^{l-1}\left\|\phi_{j, k}\right\|_{W_{0}^{l-j-1, r\left(\Omega^{\prime}\right)}}}=+\infty .
$$

The only possible way that (30) can hold, in view of the fact that the sequence $\left\|\mathcal{R}\left(\Phi_{k}\right)\right\|_{W^{l, r}(\Omega)}$ is certainly bounded, is if the denominator tends to zero. This is equivalent to saying that $\Phi_{k}$ tends to zero strongly in $\mathscr{W}_{0}^{l-1, r}\left(\Omega^{\prime}\right) \times \cdots \times \mathscr{W}_{0}^{0, r}\left(\Omega^{\prime}\right)$ which by (28) implies that $\left\|\Omega\left(\Phi_{k}\right)\right\|_{\mathscr{W}^{l, r}(\Omega)}$ tends to zero. But in this case the numerator will be negative for large values of $k$ so that (30) can not possibly hold. This completes the proof of Lemma 3.

The next step is to extend the construction to 'smooth' bounded domains. In order to do this we must first introduce some new terminology. 
Definition 3. Let $\Omega$ be a bounded domain in $\mathscr{R}^{n+1} . \Omega$ is called a bounded $\mathscr{C}^{\infty}$-domain if for every point $x_{0}$ on $\partial \Omega$ there is a neighborhood of $x_{0}$ in which $\partial \Omega$ can be represented in the form $x^{i}=f\left(x^{1}, \cdots, x^{i-1}, x^{i+1}, \cdots, x^{n+1}\right)$ for some value of $i$, where $f$ is a $C^{\infty}$-function. We may assume without loss of generality that $i=n+1$ and define variables $(y, t)$ by the equations

$$
\begin{aligned}
y^{1} & =c^{1}\left(x^{1}-x_{0}^{1}\right) \\
\vdots & \\
y^{n} & =c^{n}\left(x^{n}-x_{0}^{n}\right) \\
t & =c^{n+1}\left\{x^{n+1}-f\left(x^{1}, \cdots, x^{n}\right)\right\}
\end{aligned}
$$

where the $c$ 's are nonzero constants. Taking $c^{1}=c^{2}=\cdots=c^{n+1}=1$, the inverse function theorem tells us that on some neighborhood of $x_{0}, H$, the equations (31) define a $1-1 \mathscr{C}^{\infty}$-mapping which has a $\mathscr{C}^{\infty}$-inverse. For a suitable choice of the constants $c^{1}, \cdots, c^{n+1}$ there will then exist a neighborhood $G$ of $x_{0}$ such that $\bar{G} \subset H$, and the mapping (31) carries $G \cap \Omega$ onto $\left\{|y|^{2}+t^{2}<1 ; t>0\right\}$ and $G \cap \partial \Omega$ onto $\{|y|<1 ; t=0\}$. Furthermore there will exist another neighborhood $\frac{1}{2} G \subset G$ such that the mapping (31) carries $\frac{1}{2} G \cap \Omega$ onto $\left\{|y|^{2}+t^{2}<\frac{1}{4} ; t>0\right\}$ and $\frac{1}{2} G \cap \partial \Omega$ onto $\left\{|y|<\frac{1}{2}: t=0\right\}$.

We assume that a fixed finite collection of such neighborhoods $G_{1}, \cdots, G_{N}$ has been chosen such that the smaller neighborhoods $\frac{1}{2} G_{1}, \cdots, \frac{1}{2} G_{N}$ cover $\partial \Omega$. The corresponding transformations $T_{1}, \cdots, T_{N}$ are also assumed to be chosen and fixed.

If $\phi=\phi(x)$ is a function defined on $\partial \Omega$ then for $x$ in $G_{i} \cap \partial \Omega$ we may form $\phi\left(T_{i}^{-1} y\right)$. We now define the space $\mathscr{C}^{\infty}(\partial \Omega)$ to be the space of all functions $\phi(x)$ such that $\phi\left(T_{i}^{-1} y\right)$ is in $\mathscr{C}^{\infty}(|y|<1)$ for all $i$. We define the spaces $\mathscr{L}^{r}(\partial \Omega)$ and $\mathscr{W}^{l, r}(\partial \Omega)$ to be the spaces of all functions $\phi(x)$ such that the respective norms

and

$$
\|\phi\|_{\mathscr{L r}(\partial \Omega)}=\sum_{i=1}^{N}\left\|\phi\left(T_{i}^{-1} y\right)\right\|_{\mathscr{L r}(|y|<1)}
$$

$$
\|\phi\|_{\mathcal{W}^{l, r(\partial \Omega)}}=\sum_{i=1}^{N}\left\|\phi\left(T_{i}^{-1} y\right)\right\|_{\mathcal{W}^{l, r}(|y|<1)}
$$

make sense and are finite.

Lemma 4 (Construction in a Bounded domain). Let $\Omega$ be a bounded $\mathscr{C}^{\infty}$ domain. Let $\Phi(x)=\left\{\phi_{0}(x), \cdots, \phi_{l-1}(x)\right\}$ be a set of functions defined on $\partial \Omega$ and in the space $\mathscr{W}^{l, r}(\partial \Omega) \times \cdots \times \mathscr{W}^{1, r}(\partial \Omega) \quad(1 \leqq r<\infty)$. Then there exists a transformation $\subseteq$ such that

(i) $\subseteq$ is a linear transformation of $\mathscr{C}^{\infty}(\partial \Omega)$ into $\mathscr{C}^{\infty}(\bar{\Omega})$ and $\subseteq(\Phi)$ then assumes the Dirichlet data $\Phi(x)$ on $\partial \Omega$ i.e. $D_{s}^{j} u(x)=\phi(x)$ on $\partial \Omega$, where $D_{s}$ denotes differentiation along the inner normal to $\partial \Omega$. 
(ii) $\mathfrak{S}$ is a linear transformation of $\mathscr{W}^{l, r}(\partial \Omega) \times \cdots \times \mathscr{W}^{1, r}(\partial \Omega)$ into $\mathscr{C}^{\infty}(\Omega)$ and a compact linear transformation of $\mathscr{W}^{l, r}(\partial \Omega) \times \cdots \times \mathscr{W}^{1, r}(\partial \Omega)$ into $\mathscr{W}^{l, r}(\Omega)$.

(iii) There is a function $C(\varepsilon)$ defined for all $\varepsilon>0$ and depending only on $r$ such that

$$
\|\Im(\Phi)\|_{W^{l, r}(\Omega)} \leqq \sum_{j=0}^{l-1}\left\{\varepsilon\left\|\phi_{j}\right\|_{W^{l-j, r}(\partial \Omega)}+C(\varepsilon)\left\|\phi_{j}\right\|_{W^{l-j-1, r(\hat{r} \Omega)}}\right\}
$$

for all $\Phi(x)$ in $\mathscr{W}^{l, r}(\partial \Omega) \times \cdots \times \mathscr{W}^{1, r}(\partial \Omega)$.

(iv) If $\Phi_{k}(x)$ is a sequence which is bounded in $\mathscr{W}^{l, r}(\partial \Omega) \times \cdots \times \mathscr{W}^{1, r}(\partial \Omega)$ and strongly convergent to zero in $\mathscr{W}^{l-1, r}(\partial \Omega) \times \cdots \times \mathscr{W}^{0, r}(\partial \Omega)$ then $\Xi\left(\Phi_{k}\right)$ is strongly convergent to zero in $\mathscr{W}^{l, r}(\Omega)$.

Proof. Let $\psi_{1}(x), \cdots, \psi_{M}(x)$ be a $\mathscr{C}^{\infty}$-partition of unity chosen so that $\sum_{i=1}^{M} \psi_{i}(x) \equiv 1$ on $\Omega$ and if supp $\psi_{i} \cap \partial \Omega \neq Z$ (empty set) then supp $\psi_{i} \subset \frac{1}{2} G_{k}$ for some value of $k$. For the sake of computation we introduce a fictitious function $v=v(x)$ which 'assumes' the Dirichlet data $\Phi(x)$ on $\partial \Omega$. We rewrite $v(x)$ as $v(x)=\sum_{i=1}^{M} \psi_{i}(x) v(x)$. If $\left(\operatorname{supp} \psi_{i} \cap \partial \Omega\right) \neq Z$ then supp $\psi_{i} v \subset \frac{1}{2} G_{k_{i}}$. If we now apply the mapping $T_{k_{i}}, \psi_{i} v$ becomes a function of $(y, t)$ with support in $\left\{|y|^{2}\right.$ $\left.+t^{2}<\frac{1}{4}\right\}$ and Dirichlet data $\Phi_{i}^{\prime}$ on $t=0$.

Let $\zeta=\zeta(y, t)$ be a function in $\mathscr{C}^{\infty}\left(\mathscr{R}^{n+1}\right)$ such that $\zeta(y, t) \equiv 1$ for $|y|^{2}+t^{2} \geqq 1$. We then form

$$
\zeta \cdot \Re\left(\Phi_{i}\right)
$$

$\Re$ being the transformation given in Lemma 3. For functions $\Phi^{\prime}$ with support in $\left\{|y|^{2}+t^{2}<\frac{1}{4}\right\}$ the transformation $\zeta \cdot \Omega$ has all the properties of $\Omega$ and in addition maps $\Phi^{\prime}$ into a function which vanishes for $|y|^{2}+t^{2} \geqq 1, t>0$. In particular we have

$$
\begin{aligned}
\left\|\zeta \Re\left(\Phi_{i}\right)\right\|_{W^{l, r}\left(\mathscr{R}_{+}^{n+1}\right)} & \\
& \leqq \sum_{j=0}^{l-1}\left\{\varepsilon\left\|\phi_{j, i}^{\prime}\right\|_{W_{0}^{l-J, r\left(\mathscr{R}^{n}\right)}}+C(\varepsilon)\left\|\phi_{j, i}^{\prime}\right\|_{\left.W^{l-j-1, r\left(\mathfrak{R}^{n}\right)}\right\} .}\right.
\end{aligned}
$$

Furthermore it is clear that

$$
\left\|\phi_{j, i}^{\prime}\right\|_{W^{a, r\left(g^{n}\right)}} \leqq C \sum_{m=0}^{j+q}\left\|\phi_{m}\right\|_{W^{j+q-m, r}(\partial \Omega)} \quad(0 \leqq q \leqq l-j)
$$

where $C$ can be chosen to depend only on $l$ and $r$. Mapping back via $T_{k_{i}}^{-1}$ transforms the function $\zeta \Omega\left(\Phi_{i}\right)$ into a function $u_{i}(x)$ defined on $G_{k_{i}} \cap \Omega$. If we extend $u_{i}(x)$ to all of $\Omega$ by defining $u_{i}(x)=0$ for $x$ not in $G_{k_{i}}$ we then have $u_{i}$ in $\mathscr{W}^{l, r}(\Omega)$ and

$$
\left\|u_{i}\right\|_{W^{l, r}(\Omega)} \leqq C\left\|\zeta \Omega\left(\Phi_{i}^{\prime}\right)\right\|_{W^{l, r}\left(\mathscr{R}_{+}^{n+1}\right)}
$$

We have defined the function $u_{i}(x)$ in case supp $\psi_{i} \cap \partial \Omega \neq Z$. If supp $\psi_{i} \cap \partial \Omega=Z$ we simply set $u_{i}(x) \equiv 0$. Now define 


$$
\Im(\Phi)=\sum_{i=1}^{M} u_{i}(x)
$$

Careful checking through the steps of the construction shows that statements (i) and (ii) hold. (iii) follows from the above inequalities while (iv) is an immediate consequence of (iii).

I now give an application of Lemma 4 to the estimation of solutions of partial differential systems arising from variational problems. To state the result we give a definition of how a function assumes boundary data $\Phi(x)$ defined on $\partial \Omega$. The definition is somewhat artificial and raises some questions which we do not go into here.

Let $\Omega$ be a bounded $\mathscr{C}^{\infty}$-domain. Let $\subseteq$ be a fixed transformation of the type guaranteed by Lemma 4 . Let $\Phi(x)$ be in $\mathscr{W}^{l, r}(\partial \Omega) \times \cdots \times \mathscr{W}^{1, r}(\partial \Omega)$. We say a function $u=u(x)$ in $\mathscr{W}^{l, r}(\Omega)$ assumes the Dirichlet data $\Phi(x)$ if $u-S(\Phi)$ is in $\mathscr{W}_{0}^{l, r}(\Omega)$.

THEOREM 3. Let $f=f\left(p^{l}\right)$ be a continuously differentiable convex integrand such that

$$
K_{1}\left|p^{l}\right| r-K_{1}^{\prime} \leqq f\left(p^{l}\right) \leqq K_{2}\left|p^{l}\right|^{r}+K_{2}^{\prime} \quad(1 \leqq r<\infty)
$$

where $K_{1}$ is a positive constant. Let $F(u)=0$ be the Euler equation for the variational problem

$$
\underset{u \operatorname{in} \mathscr{W}^{l}(\Omega)(\Omega)}{\operatorname{Min}} \int_{\Omega} f\left(D^{l} u(x)\right) d x
$$

We conclude that if $u(x)$ is in $\mathscr{W}^{l, r}(\Omega)$ and is a weak solution of $F(u)=0$ assuming Dirichlet data $\Phi(x)$ on $\partial \Omega$ in the sense given above then

$$
\begin{aligned}
\|u\|_{W^{l, r}(\Omega)} \leqq & \left(1+\left(\frac{K_{2}}{K_{1}}\right)^{1 / r}\right)_{j=0}^{l-1}\left\{\varepsilon\left\|\phi_{j}\right\|_{W^{l-j, r(\partial \Omega)}}+C(\varepsilon)\left\|\phi_{j}\right\|_{\left.W^{l-j-1, r(\partial \Omega)}\right\}}\right\} \\
& +C^{\prime}\left(\frac{K_{1}^{\prime}+K_{2}^{\prime}}{K_{1}}\right)^{1 / r}(\operatorname{meas} \Omega)^{1 / r},
\end{aligned}
$$

where $C(\varepsilon)$ and $C^{\prime}$ depend only on $l$ and $r$. In particular if $K_{1}^{\prime}=K_{2}^{\prime}=0$ and $u_{k}(x)$ is a sequence of solutions with Dirichlet data $\Phi_{k}(x)$ such that $\Phi_{k}(x)$ is bounded in $\mathscr{W}^{l}(\partial \Omega) \times \cdots \times \mathscr{W}^{1, r}(\partial \Omega)$ and strongly convergent to zero in $\mathscr{W}^{l-1, r}(\partial \Omega) \times \cdots \times \mathscr{W}^{0, r}(\partial \Omega)$ then $u_{k}(x)$ is strongly convergent to zero in $\mathscr{W}^{l, r}(\Omega)$.

Proof. Since $u(x)$ solves the Euler equations and since the integrand $f\left(P^{l}\right)$ is convex it easily follows that $u(x)$ is a solution of the variational problem

$$
\underset{u \text { in } \mathscr{D}}{\operatorname{Min}} \int_{\Omega} f\left(D^{l} u(x)\right) d x
$$


where $\mathscr{D}$ is the Dirichlet class of $\mathscr{W}^{l, r}(\Omega)$ containing $\Theta(\Phi)$. Letting $\circlearrowleft(\Phi)=v$ and denoting the above variational integral by $I$ we then have

$$
K_{1}\left\|D^{l} u\right\|_{\mathscr{L}^{r}(\Omega)}^{r}-K_{1}^{\prime} \text { meas } \Omega \leqq I(u) \leqq I(v) \leqq K_{2}\left\|D^{l} v\right\|_{\mathscr{L}^{r}(\Omega)}^{r}+K_{2}^{\prime} \text { meas } \Omega .
$$

Therefore

$$
\left\|D^{l} u\right\|_{\mathscr{L} r(\Omega)} \leqq\left(\frac{K_{2}}{K_{1}}\right)^{1 / r}\left\|D^{l} v\right\|_{\mathscr{L}(\Omega)}+\left(\frac{K_{1}^{\prime}+K_{2}^{\prime}}{K_{1}}\right)^{1 / r}(\operatorname{meas} \Omega)^{1 / r} .
$$

Since $u(x)-v(x)=w(x)$ is in $\mathscr{W}_{0}^{l, r}(\Omega)$ it is well known that

Therefore

$$
\|w\|_{W^{l-1, r}(\Omega)} \leqq C\left\|D^{l} w\right\|_{\mathscr{L} r(\Omega)} .
$$

$$
\|u\|_{\mathscr{W}^{l-1, r(\Omega)}} \leqq C\left\{\|v\|_{\mathscr{W}^{l, r}(\Omega)}+\left\|D^{l} u\right\|_{\mathscr{L}(\Omega)}\right\}
$$

From (32) and (33) we then have

$$
\|u\|_{W^{\operatorname{lir}(\Omega)}} \leqq C\left(1+\left(\frac{K_{2}}{K_{1}}\right)^{1 / r}\right)\|v\|_{W^{\operatorname{lor}(\Omega)}}+\left(\frac{K_{1}^{\prime}+K_{2}^{\prime}}{K_{1}}\right)^{1 / r}(\operatorname{meas} \Omega)^{1 / r} .
$$

Since $v(x)$ satisfies (iii) of Lemma 4, the desired inequality easily follows. The last statement is an immediate consequence of the inequality.

4. Lower semi-continuity with respect to weak convergence in $\mathscr{W}^{l, r}(\Omega)$ $(1 \leqq r<\infty)$. We first define the basic class of integrands $f\left(x, p^{l}\right)$ to be covered by the theory.

Definition 4. Let $\Omega$ be a bounded domain in $\mathscr{R}^{n}$. A continuous integrand $f\left(x, p^{l}\right)$ is said to be in the class $\mathscr{T}_{r}(\Omega)(1 \leqq r<\infty)$ if

(i) $f\left(x, p^{l}\right) \leqq C\left\{1+\left|p^{l}\right|\right\}^{r}$

where $C$ is a constant,

(ii) $\left|f\left(x, p^{l}+q^{l}\right)-f\left(x, p^{l}\right)\right| \leqq C\left\{1+\left|p^{l}\right|+\left|q^{l}\right|\right\}^{r-\gamma} \cdot\left|q^{l}\right|^{\gamma}$

where $C$ is a constant and $\gamma$ is a constant, $0<\gamma \leqq 1$,

(iii) $\left|f\left(x+y, p^{l}\right)-f\left(x, p^{l}\right)\right| \leqq\left\{1+\left|p^{l}\right|\right\}^{r} \cdot \eta(|y|)$

where $\eta$ is a continuous increasing function with $\eta(0)=0$.

Lemma 5. Let $\Omega$ be a bounded $C^{\infty}$-domain, $f\left(x, p^{l}\right)$ an integrand in $\mathscr{T}_{r}(\Omega)$ and $I(u)$ its corresponding integral defined for all $u(x)$ in $\mathscr{W}^{l, r}(\Omega)$. Let $z_{k}(x)$ be a sequence of functions in $\mathscr{C}^{\infty}(\bar{\Omega})$ which is weakly convergent in $\mathscr{W}^{l, r}(\Omega)$ and whose Dirichlet data, $\Phi_{k}(x)$, on $\partial \Omega$ satisfy:

(i) the set of $\Phi_{k}$ is bounded in $\mathscr{W}^{l, r}(\partial \Omega) \times \cdots \times \mathscr{W}^{1, r}(\partial \Omega)$,

(ii) $\Phi_{k} \stackrel{s}{\rightarrow} 0$ in $\mathscr{W}^{l-1, r}(\partial \Omega) \times \cdots \times \mathscr{W}^{0, r}(\partial \Omega)$.

If $\mathscr{D}$ is a Dirichlet class of $\mathscr{W}^{l, r}(\Omega)$ and if $I / \mathscr{D}$ attains an absolute maximum at $w(x)$ then

$$
\liminf _{k \rightarrow \infty} I\left(w+z_{k}\right) \geqq I(w) .
$$


Proof. According to Lemma 4 there exists a sequence $u_{k}(x)$ in $C^{\infty}(\bar{\Omega})$ such that $u_{k}(x)-z_{k}(x)$ is in $\mathscr{W}_{0}^{l, r}(\Omega)$ and such that $u_{k}(x) \stackrel{s}{\rightarrow} 0$ in $\mathscr{W}^{l, r}(\Omega)$ as $k \rightarrow \infty$. Rewrite

$$
\begin{aligned}
I\left(w+z_{k}\right)-I(w) & =\left\{I\left(w+z_{k}\right)-I\left(w+z_{k}-u_{k}\right)\right\}+\left\{I\left(w+z_{k}-u_{k}\right)-I(w)\right\} \\
& =J_{1}+J_{2} .
\end{aligned}
$$

Using property (ii) of Definition 4 and the Hölder inequality we get

$$
J_{1} \leqq C\left\{1+\left\|w+z_{k}\right\|_{W^{l, r}(\Omega)}+\left\|u_{k}\right\|_{W^{l, r}(\Omega)}\right\}^{r-\gamma}\left\|u_{k}\right\|_{W^{l, r}(\Omega)}^{\gamma} .
$$

Since the sequence $z_{k}(x)$ is weakly convergent the quantity in the brackets is bounded independent of $k$ and from the strong convergence of $u_{k}(x)$ we have

$$
\underset{k \rightarrow \infty}{J_{1} \rightarrow 0}
$$

From the assumptions of the lemma

$$
J_{2} \geqq 0,
$$

and the result follows immediately from (34) and (35).

LEMMA 6. Let $I=I(u)$ be a real-valued functional defined on a normed linear space $\mathscr{W}$ and let $I(u)$ be continuous relative to strong convergence. Let $\Delta$ be a dense subset (in the strong topology) of the linear space such that 0 is in $\Delta$. Then, if $I / \Delta$ is lower semi-continuous at 0 relative to weak convergence, it is also true of $I$.

Proof. Let $u_{k}$ be a sequence in $\mathscr{W}$ such that $u_{k} \stackrel{w}{\rightarrow} 0$ as $k \rightarrow \infty$. Let $v_{k}$ be a corresponding sequence in $\Delta$ chosen so that

and

$$
u_{k}-v_{k} \stackrel{s}{\rightarrow} 0 \text { as } k \rightarrow \infty
$$

$$
I\left(u_{k}\right)-I\left(v_{k}\right) \geqq-\frac{1}{k} \text { for each } k \text {. }
$$

This is certainly possible under our assumptions. Hence $v_{k} \stackrel{w}{\rightarrow} 0$ as $k \rightarrow \infty$ so that

$$
\lim _{k \rightarrow \infty} \inf I\left(v_{k}\right) \geqq I(0),
$$

and it follows easily from the above that $\lim \inf I\left(u_{k}\right) \geqq I(0)$ as $k \rightarrow \infty$.

Lemma 6 permits us to use dense sets of elements when testing for lower semicontinuity. Lemma 7 which follows, will permit us to use 'smooth' functions in $\mathscr{W}^{l, r}(\Omega)$ when testing for lower semi-continuity. A proof of Lemma 7 which was recently given by Professor James Serrin and myself can be found in [2].

LEMMA 7. The subspace $\mathscr{C}^{\infty}(\Omega) \cap \mathscr{W}^{l, r}(\Omega)(1 \leqq r<\infty)$ is dense (in the strong topology) in $\mathscr{W}^{l, r}(\Omega)$.

THEOREM 4. Let $\Omega$ be a bounded domain in $\mathscr{R}^{n}$ and let $f\left(x, p^{l}\right)$ be an integrand 
in the class $\mathscr{T}_{r}(\Omega)(1 \leqq r<\infty)$ and $I(u)$ its corresponding integral which is defined for all $u(x)$ in $\mathscr{W}^{l, r}(\Omega)$. Let $\mathscr{D}$ be either a Dirichlet class in $\mathscr{W}^{l, r}(\Omega)$ or $\mathscr{W}^{l, r}(\Omega)$ itself. Then $I / \mathscr{D}$ is lower semi-continuous relative to weak convergence in $\mathscr{W}^{l, r}(\Omega)$ if and only if

(i) $f\left(x, p^{l-1}, p^{l}\right)$ is quasi-convex in $p^{l}$ for each fixed value of $\left(s, p^{l-1}\right)$,

(ii) $\lim \inf _{k \rightarrow \infty} I\left(u_{k} ; \Omega^{\prime}\right) \geqq-\mu$ (meas $\left.\Omega^{\prime}\right)$ for every subdomain $\Omega^{\prime}$ and every sequence $u_{k}(x)$ in $\mathscr{D}$ such that $u_{k}(x) \equiv u(x)$ on $\Omega-\Omega^{\prime}$ and $u_{k}(x) \stackrel{w}{\rightarrow} u(x)$ in $\mathscr{W}^{l, r}(\Omega) ; \mu$ is a continuous increasing function with $\mu(0)=0$ and depends only on the function $u(x)$ and on $\lim \sup _{k \rightarrow \infty}\left\|u_{k}\right\|_{w^{l i r}(\Omega)}\left({ }^{(2)}\right.$

Proof. (Necessity) We first prove the necessity of (i), the quasi-convexity condition. Let $x_{1}$ be an arbitrary point in $\Omega$ and let $B$ be an open ball with center $x_{1}$ such that $\bar{B} \subset \Omega$. Let $\psi(x)$ be a function in $\mathscr{C}_{0}^{\infty}(\Omega)$ which is identically equal to 1 on $B$. Lastly, let $v(x)$ be a fixed function in $\mathscr{D}$ and $u_{k}(x), u(x)$ functions in $\mathscr{W}_{0}^{l}{ }^{\infty}(B)$ such that

$$
u_{k \rightarrow \infty} \rightarrow u \text { in } \mathscr{W}^{l, \infty}(B)
$$

The functions $\psi u_{k}+(1-\psi) v$ and $\psi u+(1-\psi) v$ are in $\mathscr{D}$ and $\psi u_{k}+(1-\psi) v$ $\stackrel{w}{\rightarrow} \psi u+(1-\psi) v$ in $\mathscr{W}^{l, r}(\Omega)$. Hence

$$
\liminf _{k \rightarrow \infty} I\left(\psi u_{k}+(1-\psi) v\right) \geqq I(\psi u+(1-\psi) v) .
$$

Since $I\left(\psi u_{k}+(1-\psi) v\right)-I(\psi u+(1-\psi) v)=I\left(u_{k} ; B\right)-I(u ; B)$, it follows from (36) that $I(u ; B) / \mathscr{W}_{0}^{l, \infty}(B)$ is lower semi-continuous with respect to convergence in $\mathscr{W}^{l, \infty}(B)$. Therefore from Theorem 1 we have that $f\left(x_{1}, p^{l-1}, p^{l}\right)$ is quasiconvex in $p^{l}$ for each fixed value of $p^{l-1}$ and since $x_{1}$ is arbitrary (i) is proved.

We next prove the necessity of condition (ii). Using the notation of the statement of (ii), we know

$$
\liminf _{k \rightarrow \infty} I\left(u_{k} ; \Omega\right) \geqq I(u ; \Omega) .
$$

Since $u_{k}(x) \equiv u(x)$ on $\Omega-\Omega^{\prime}$, this implies

$$
\underset{k \rightarrow \infty}{\lim \inf } I\left(u_{k} ; \Omega^{\prime}\right) \geqq I\left(u ; \Omega^{\prime}\right) .
$$

Now $I\left(u ; \Omega^{\prime}\right)$ is an absolutely continuous set function, so the result follows from (37). In fact we have proved a stronger necessary condition than (ii) in that $\mu$ does not depend on $\lim \sup _{k \rightarrow \infty}\left\|u_{k}\right\|_{W^{\prime} \text { ir }(\Omega)}$ and $\Omega^{\prime}$ can be replaced by any measurable subset of $\Omega$.

$\left(^{2}\right)$ If we change the assumption in (ii) to $u_{k}(x)$ is bounded in $\mathscr{W}_{i, r}(\Omega)$ and $u_{k}(x) \stackrel{s}{\rightarrow} u(x)$ in $\mathscr{W}^{i-1, r}(\Omega)$, inspection of the proofs will show that $1 / \mathscr{D}$ is lower semi-continuous under this type of convergence. In case $1<r<\infty$ this is equivalent to weak convergence due to the reflexivity of the space; but if $1=r$ it is weaker than weak convergence. Note that this mode of convergence is also the direct analog of the convergence we considered for $r=\infty$. 
(Sufficiency) Let $u(x)$ be a function in $\mathscr{D}$. We wish to test for the lower semicontinuity of $I / \mathscr{D}$ at $u(x)$. We therefore consider a sequence $u(x)+z_{k}(x)$, where $z_{k}(x)$ is in $\mathscr{W}_{0}^{l, r}(\Omega)$ if $\mathscr{D}$ is a Dirichlet class and $z_{k}(x)$ is merely in $\mathscr{W}^{l, r}(\Omega)$ if $\mathscr{D}=\mathscr{W}^{l, r}(\Omega)$, and in either case $z_{k}(x) \stackrel{w}{\rightarrow} 0$ in $\mathscr{W}^{l, r}(\Omega)$. If we set $J(z)=I(u+z)$ and apply Lemmas 6 and 7 to the functional $J$, it becomes clear that it suffices to take $z_{k}(x)$ in $\mathscr{C}_{0}^{\infty}(\Omega)$ if $\mathscr{D}$ is a Dirichlet class and $z_{k}(x)$ in $\mathscr{C}^{\infty}(\Omega)$ if $\mathscr{D}=\mathscr{W}^{l, r}(\Omega)$.

As in the proof of Theorem 2, let $G_{v}(v=1,2, \cdots)$ be the cubic lattice containing the cube $0 \leqq x^{i} \leqq 2^{-v}$ and let $\Gamma_{v}$ be the union of those cubes which are contained in $\Omega$. If $\delta>0$ then for some sufficiently large value of $v=v^{\prime}$

$$
\text { meas }\left(\Omega-\Gamma_{v^{\prime}}\right)<\delta \text {. }
$$

Let $v \geqq v^{\prime}$ and corresponding to each such $v$ choose a finite set of open $n$-balls $B_{v, 1}, \cdots, B_{v, N}$ such that

(39) for each $v$ the $B_{v, i}$ have disjoint closures and each $B_{v, i}$ is contained in a lattice cube of $G_{v}$ which is contained in $\Gamma_{v^{\prime}}$ and

$$
\text { meas }\left(\Gamma_{v^{\prime}}-B_{v}\right)<\delta
$$

where $B_{v}=\sum_{i=1}^{N_{v}} B_{v, i}$. Since the functions $z_{k}(x) \stackrel{w}{\rightarrow} 0$ in $\mathscr{W}^{l, r}(\Omega)$, it follows by standard procedures, that after a possibly arbitrarily small decrease in the radii (the centers are left fixed) of some of the balls $B_{v, i}$ one can in addition to (39) and (40) satisfy the condition, some subsequence of the $z_{k}(x)$ exists (call it $z_{k_{h}}(x)$ with Dirichlet data $\Phi_{k_{h}}(x)$ on $\left.B_{v}\right)$ such that

$$
\begin{aligned}
& \Phi_{k_{h}}(x) \text { is bounded in } \mathscr{W}^{l, r}\left(\partial B_{v, i}\right) \times \cdots \times \mathscr{W}^{1, r}\left(\partial B_{v, i}\right), \\
& \Phi_{k_{h}}(x) \underset{h \rightarrow \infty}{\stackrel{s}{\rightarrow}} 0 \text { in } \mathscr{W}^{l-1, r}\left(\partial B_{v, i}\right) \times \cdots \times \mathscr{W}^{0, r}\left(\partial B_{v, i}\right)
\end{aligned}
$$

for each $v$ and $i$. (For a proof see the Appendix.) Now

$$
\begin{aligned}
I(u+ & \left.z_{k} ; B_{v}\right)-I\left(u ; B_{v}\right) \\
= & \sum_{i=1}^{N_{v}} \int_{B_{v}, i}\left\{f\left(x, D^{l} u(x)+D^{l} z_{k}(x)\right)-f\left(\bar{x}, D^{l} u(x)+D^{l} z_{k}(x)\right)\right\} d x \\
& +\sum_{i=1}^{N_{v}} \int_{B_{v}, i}\left\{f\left(\bar{x}, D^{l} u(x)\right)-f\left(x, D^{l} u(x)\right)\right\} d x \\
& +\int_{B_{v}}\left\{f\left(\bar{x}, D^{l} u(x)+D^{l} z_{k}(x)\right)-f\left(\bar{x}, \overline{D^{l} u(x)}+D^{l} z_{k}(x)\right)\right\} d x \\
& +\int_{B_{v}}\left\{f\left(\bar{x}, \overline{D^{l}} u(x)\right)-f\left(\bar{x}, D^{l} u(x)\right)\right\} d x \\
& +\sum_{i=1}^{N_{v}} \int_{B_{v}, i}\left\{f\left(\bar{x}, \overline{D^{l} u} u(x)+D^{l} z_{k}(x)\right)-f\left(\bar{x}, \overline{D^{l} u} u(x)\right)\right\} d x \\
= & J_{1}+\cdots+J_{5} .
\end{aligned}
$$


Here, as in the proof of Theorem 2, if $v(x)$ is in $\mathscr{L}^{r}\left(\Gamma_{v^{\prime}}\right)$ then $\bar{v}(x)$ is a new function defined by

$$
\bar{v}(x)=(\operatorname{meas} Q)^{-1} \int_{Q} v(x) d x
$$

for $x$ in the interior of the cube $Q$ of the lattice $G_{v}\left(v \geqq v^{\prime}\right)$. It is well known and not hard to prove that

$$
\|\bar{v}\|_{\mathscr{L} r^{(}\left(\Gamma_{v^{\prime}}\right)} \leqq\|v\|_{\mathscr{L} r\left(\Gamma_{v^{\prime}}\right)}
$$

and

$$
\bar{v} \stackrel{s}{\rightarrow} v \text { in } \mathscr{L}^{r}\left(\Gamma_{v^{\prime}}\right) \text { as } v \rightarrow \infty .
$$

Returning to the proof, we see that from condition (iii) of Definition 4 and the weak convergence of the $z_{k}(x)$ that

$$
J_{1} \underset{v \rightarrow \infty}{\rightarrow} 0 \text { and } J_{2} \underset{v \rightarrow \infty}{\rightarrow} 0 \text { uniformly in } k .
$$

As for $J_{3}$ note that

$$
J_{3} \leqq \int_{\Gamma_{v^{\prime}}}\left|f\left(\bar{x}, D^{l} u(x)+D^{l} z_{k}(x)\right)-f\left(\bar{x}, \overline{D^{l}} u(x)+D^{l} z_{k}(x)\right)\right| d x,
$$

which from condition (ii) of Definition 4, leads to

$$
\left.J_{3} \leqq C\left\{1+\|u\|_{\mathscr{W}^{l}, r(\Omega)}+\left\|z_{k}\right\|_{\mathscr{W}^{l, r}(\Omega)}+\left\|\overline{D^{l} u}\right\|_{\mathscr{L}^{r}\left(\Gamma_{v},\right.}\right)\right\}^{r-\gamma}\left\|D^{l} u-\overline{D^{\prime} u}\right\|_{\mathscr{L}^{r}\left(\Gamma_{v^{\prime}}\right)}^{\gamma} .
$$

From the weak convergence of the $z_{k}(x)$ and from (43), (44) we easily deduce

$$
\underset{v \rightarrow \infty}{J_{3} \rightarrow 0} \text { uniformly in } k,
$$

and since the same argument applies equally to $J_{4}$,

$$
\underset{v \rightarrow \infty}{J_{4} \rightarrow 0} \text { uniformly in } k \text {. }
$$

Now we turn to the all important integral $J_{5}$. Recalling that at least for some subsequence of the $z_{k}(x)$ condition (41) holds, it follows from the quasi-convexity assumption and from Lemma 8 that

$$
\lim _{h \rightarrow \infty} J_{5} \geqq 0 \text { for each } v \geqq v^{\prime}
$$

where the lim inf in (48) is taken over the subsequence of (41). Hence from (45)-(48), for each $\varepsilon>0$ there exists a value of $v$ such that

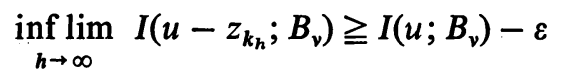

for the subsequence satisfying (41). 
We have still not used condition (ii) of the theorem. We now use it. Fixing $v$ at a value for which (49) holds, construct auxiliary functions $v_{h}(x)$ in $\mathscr{C}^{\infty}\left(\Omega-B_{v}\right)$ such that

(i') $z_{k_{h}}-v_{h} \stackrel{s}{\rightarrow} 0$ in $\mathscr{W}^{l, r}\left(\Omega-\bar{B}_{v}\right)$ as $h \rightarrow \infty$.

(ii') $D^{l-1} v_{h}(x)=0$ on $\partial B_{v}$.

(iii') $z_{k_{h}}(x) \equiv v_{h}(x)$ in some fixed neighborhood of $\partial \Omega$ independent of $h$.

The existence of the $v_{h}(x)$ follows from Lemma 4 by a simple argument. Now extend the functions $v_{h}(x)$ to all of $\Omega$ by defining them to be identically zero on $B_{v}$. Then $v_{h}(x)$ is in $\mathscr{D}$. From (i') above and (ii) of Definition 4 we have

$$
\lim _{h \rightarrow \infty}\left\{I\left(u+z_{k_{h}} ; \Omega-B_{v}\right)-I\left(u+v_{h} ; \Omega-B_{v}\right)\right\}=0 .
$$

From condition (ii) of this theorem and (38), (40)

$$
\lim _{h \rightarrow \infty} I\left(u+v_{h} ; \Omega-B_{v}\right) \geqq-\mu(2 \delta) .
$$

By combining (49)-(51) we conclude

$$
\underset{h \rightarrow \infty}{\lim \inf } I\left(u+z_{k_{h}} ; \Omega\right) \geqq I(u ; \Omega)-I\left(u ; \Omega-B_{v}\right)-\varepsilon-\mu(2 \delta) .
$$

Since $\varepsilon$ and $\delta$ are arbitrary and $I(u ; \Gamma)$ is an absolutely continuous set function it follows that

$$
\underset{h \rightarrow \infty}{\lim \inf } I\left(u+z_{k_{h}} ; \Omega\right) \geqq I(u ; \Omega) .
$$

Because the sequence $z_{k}(x)$ was arbitrary it follows by an obvious argument that (52) holds for the entire sequence $z_{k}(x)$, which concludes the proof.

Remarks on THEOREM 4. The condition of quasi-convexity is by its very nature difficult to verify in general. However it is well known that there is a more tractable condition of reasonably wide scope which implies quasi-convexity, namely convexity (in $p^{l}$ ). In fact, in the special case $m=1, l=1$ the two conditions are equivalent, though this is definitely not the case for higher values of $m$ and $l$, as witness the quadratic variational theory.

Unlike the condition of quasi-convexity which has nothing to do with the function class $\mathscr{D}$ that one considers, condition (ii) definitely depends on $\mathscr{D}$. For if we again take quadratic variational theory as an example, we see that even under quasi-convexity, (ii) can hold for $\mathscr{D}=\mathscr{W}_{0}^{l, r}(\Omega)$ without holding for $\mathscr{D}=\mathscr{W}^{l, r}(\Omega)$. This also illustrates independence of (i) and (ii). One should think of (ii) as determining the proper boundary condition. Like quasi-convexity it is hard to see how to go about verifying condition (ii). However again reasonably broad sufficient conditions exist. For example if $f \geqq C$ (a constant) then (ii) holds with $\mathscr{D}=\mathscr{W}^{l, r}(\Omega)$. More generally, if $f \geqq g$ where the integral for $g$ is known to be a lower semicontinuous functional on $\mathscr{D}$ then (ii) holds for $\mathscr{D}$. In fact we need only know the 
definitely weaker inequality, integral for $f \geqq$ integral for $g$ on $\mathscr{D}$. So, for example, $I / \mathscr{D} \geqq 0$ is sufficient. Another important case in which (ii) must hold is given in the following theorem where one proves lower semi-continuity without using (ii) explicitly.

THEOREM 5. Let $l=1$ and let $Q$ be a bounded open cube in $\mathscr{R}^{n}$. Let $f=f\left(x, p^{0}, p^{1}\right)$ be in the class $\mathscr{T}_{r}(Q)(1 \leqq r<\infty)$ and quasi-convex in $p_{1}$. If $\Omega$ is a subdomain of $Q$ and $\mathscr{D}$ is a Dirichlet class of $\mathscr{W}^{l, r}(\Omega)$ which contains a function in $\mathscr{W}^{l, r}(Q)$, then $I / \mathscr{D}$ is lower semi-continuous with respect to weak convergence in $\mathscr{W}^{l, r}(\Omega)$.

Proof. We shall only outline the proof since the main ideas have already been covered and occur also in [3]. We first prove the theorem in the special case $\Omega=Q$. As before it suffices to consider the case $u(x)+z_{k}(x) \stackrel{w}{\rightarrow} u(x)$ in $\mathscr{W}^{1, r}(Q)$ where $z_{k}(x)$ is in $\mathscr{C}_{0}^{\infty}(Q)$ and $u(x)$ is an arbitrary element of $\mathscr{D}$. Let $G_{v}(v=1,2, \cdots)$ be a sequence of cubic lattices on $Q$ whose meshes (diameter of the cubes composing $\left.G_{v}\right) \rightarrow 0$ as $v \rightarrow \infty$. Moreover, let the $G_{v}$ be chosen so that for some subsequence $z_{k_{h}}(x)$

$$
\begin{aligned}
& D z_{k_{h}}(x) \text { is bounded in } \mathscr{L}^{r}(S), \\
& z_{k_{h}}(x) \underset{h \rightarrow \infty}{\stackrel{s}{\rightarrow}} 0 \text { in } \mathscr{L}^{r}(S),
\end{aligned}
$$

where $S$ is any $(n-1)$-dimensional face of any cube in $G_{v}$. One now uses the fact that if (53) holds on each face of an open cube $Q^{\prime}$ then there is an auxiliary sequence $v_{h}(x)$ such that

$$
\begin{aligned}
& v_{h}(x)=z_{k_{h}}(x) \text { on } \partial Q^{\prime}, \\
& v_{h}(x) \stackrel{s}{\rightarrow} 0 \text { in } \mathscr{W}^{1, r}\left(Q^{\prime}\right) .
\end{aligned}
$$

This fact can be proved using the same ideas as before, that is Poisson kernels, or in a much more direct and elementary fashion as is done in [3]. The proof now proceeds as in Theorem 3 except that the open balls $B_{v, 1}, \cdots, B_{v, N}$ are replaced by the cubes of the lattice $G_{v}$.

If $\Omega \subset Q$ then again consider $u(x)+z_{k}(x) \stackrel{w}{\rightarrow} u(x)$ in $\mathscr{W}^{l, r}(\Omega)$ where $u(x)$ is in $\mathscr{D}$ and $z_{k}(x)$ is now in $\mathscr{W}_{0}^{l, r}(\Omega)$. Thus $z_{k}(x)$ can be considered in $\mathscr{W}_{0}^{l, r}(Q)$ by defining it to be identically zero outside $\Omega$ and from our assumption on $\mathscr{D}, u(x)$ has an extension to $Q$ which is in $\mathscr{W}^{l, r}(Q)$. Since

$$
I\left(u+z_{k} ; \Omega\right)-I(u ; \Omega)=I\left(u+z_{k} ; Q\right)-I(u ; Q)
$$

the result follows.

In view of Theorem 5 we are led to the following conjecture.

CONJECTURE. If condition (ii) is dropped from Theorem 4, the theorem is still true provided $\mathscr{D}$ is a Dirichlet class containing a suitably 'smooth' function or $\partial \Omega$ is suitably 'smooth'. 
Less restrictive growth conditions on the integrand. In a large number of cases it is possible to broaden the class of integrands to which Theorem 4 applies to a new class $\mathscr{T}_{r}^{*}(\Omega)(1 \leqq r<\infty)$. To define this class let

$$
J=\max \left(\text { largest integer }<l-\frac{n}{r},-1\right) .
$$

For $j=J+1, \cdots, l$ define $r_{j}$ by

$$
\frac{1}{r_{j} !}=\frac{1}{r}+\frac{j-l}{n} \text { for } j>l-\frac{n}{r}
$$

and

$$
r_{j}=\text { any number in the range } 1 \leqq r_{j}<\infty \text { for } j=l-\frac{n}{r} .
$$

Set

$$
s_{j, k}=\min \left(r_{j}, r_{k}\right) \text { for } j, k=J+1, \cdots, l .
$$

In the following, any function indicated by the generic letter $K$ is a growth modulus at infinity, i.e., $K$ is some continuous increasing function of its argument. Any function indicated by $\eta$ is a modulus of continuity, i.e., $\eta$ is some continuous increasing function with $\eta(0)=0$. We further adopt the convention that $\left|p^{-1}\right|=1$. We say that a continuous integrand $f=f\left(x, p^{l}\right)$ is in the class $\mathscr{T}_{r}^{*}(\Omega)$ if it is defined for all $x$ in $\Omega$ and all values of $p^{l}$ and satisfies

$$
\left|f\left(x, p^{l}\right)\right| \leqq K\left(\left|p^{J}\right|\right)\left\{1-\sum_{k=J+1}^{l}\left|p^{k}\right|^{r_{k}}\right\}
$$

$$
\left|f\left(x, p^{l}+q^{l}\right)-f(x, p)^{l}\right|
$$

(ii) $\leqq K_{1}\left(\left|p^{J}\right|+\left|q^{J}\right|\right)\left\{1+\sum_{k=J+1}^{l}\left(\left|p^{k}\right|+\left|q^{k}\right|\right)^{r_{k}}\right\} \cdot \eta\left(\left|q^{J}\right|\right)$

$$
+\sum_{j=J+1}^{l} K_{1}\left(\left|p^{J}\right|+\left|q^{J}\right|\right)\left\{1+\sum_{k=J+1}^{l}\left(\left|p^{k}\right|+\left|q^{k}\right|\right)^{s_{j, k}-\gamma}\right\}\left|q_{j}\right|^{\gamma}
$$

$$
(0<\gamma \leqq 1) .
$$

(iii) $\left|f\left(x+y ; p^{l}\right)-f\left(x, p^{l}\right)\right| \leqq K_{2}\left(\left|p^{J}\right|\right)\left\{1+\sum_{k=J+1}^{l}\left|p^{k}\right|^{r_{k}}\right\} \cdot \eta_{1}(|y|)$.

THEOREM 6. The conclusion of Theorem 4 remains true if the integrand $f\left(x, p^{l}\right)$ is in the class $\mathscr{T}_{r}^{*}(\Omega)$ provided that $\partial \Omega$ is a 'smooth' surface (a $\mathscr{C}^{l}$-surface is certainly sufficient) or $\mathscr{D}$ is a Dirichlet class containing a function $u(x)$ in $\mathscr{W}^{l, r}\left(\Omega^{\prime}\right)$ where $\bar{\Omega} \subset \Omega^{\prime}\left(\right.$ e.g. $\left.\mathscr{D}=\mathscr{W}_{0}^{l, r}(\Omega)\right)$.

Proof. The proof is the same as that of Theorem 4 except that one now takes into account the well-known Sobolev inequalities which in the above circumstances 
imply that if $u(x)$ is in $\mathscr{D}$ then $u(x)$ is also in $\mathscr{W}^{j, r j}(\Omega)$ for $j=J+1, \cdots, l$ and in $\mathscr{C}^{\mathrm{J}}(\bar{\Omega})$. We leave the details of proof to the reader.

5. The Legendre condition. Denote the components of $p^{l}$ by $p^{\alpha, i}$ where $|\alpha|=l$ and $i=1, \cdots, m$. Let $\xi=\left(\xi^{1}, \cdots, \xi^{n}\right)$ and $\eta=\left(\eta^{1}, \cdots, \eta^{m}\right)$ and set $\xi^{\alpha}=\left(\xi^{1}\right)^{\alpha_{1}} \cdots\left(\xi^{n}\right)^{\alpha_{n}}$.

THEOREM 7. If the integrand $f=f\left(p^{l}\right)$ is twice continuously differentiable and quasi-convex then it satisfies the Legendre condition

$$
\sum_{|\alpha|=|\beta|=l ; i, j=1, \cdots, m} \frac{\partial^{2} f\left(p^{l}\right)}{\partial p^{\alpha, i} \partial p^{\beta, j}} \xi^{\alpha} \xi^{\beta} \eta^{i} \eta^{j} \geqq 0 .
$$

Proof. Let $Q$ be the cube $0<x^{t}<1$ and let $z(x)$ be any function in $\mathscr{C}_{0}^{\infty}(Q)$. Choosing $p^{l}$ arbitrarily, set

$$
I(\varepsilon)=\int_{Q}\left\{f\left(p^{l}+\varepsilon D^{l} z(x)\right)-f\left(p^{l}\right)\right\} d x .
$$

We know that $I(\varepsilon)$ attains an absolute minimum at $\varepsilon=0$ and therefore $d^{2} I(0) / d \varepsilon^{2}$ $\geqq 0$. Carrying out the differentiation we get

$$
\int_{Q} \sum_{|\alpha|=|\beta|=l ; i, j=1, \cdots, m} \frac{\partial^{2} f\left(p^{l}\right)}{\partial p^{\alpha, i} \partial p^{\beta, j}} D^{\alpha} z^{i}(x) D^{\beta} z^{j}(x) d x \geqq 0 .
$$

But it is well known that the necessary and sufficient condition for the inequality (54) to hold for all $z(x)$ in $\mathscr{C}_{0}^{\infty}(Q)$ is that $f\left(p^{l}\right)$ satisfy the Legendre condition (see [5]).

For necessary conditions (Weierstrass condition) for quasi-convexity when $f\left(p^{l}\right)$ is not twice continuously differentiable see [3], which can all be carried over directly to the case $l>1$.

This paper was written with the support of the Office of Naval Research under project Nonr. 710 (54) NR-053-041.

\section{APPENDIX}

Proof of (41). We first consider the following situation. Let $B$ be the open ball $\{|x|<1\}$, let $g_{k}(x)$ be a sequence of functions such that

$$
\int_{B} g_{k}(x) d x \leqq M<\infty \text { for all } k
$$

and let $S$ be a subset of $(0,1)$ with linear measure equal to 1 . We prove that for each $\varepsilon, 0<\varepsilon<1$, there exists a sphere $\sigma_{\rho}=\{|x|=\rho\}$ such that $1-\varepsilon<\rho<1$, $\rho$ is in $S$ and for some subsequence 


$$
\int_{\sigma_{n}} g_{k_{h}}(x) d \sigma_{\rho} \leqq M^{\prime}<\infty \text { for all } h,
$$

$d \sigma_{\rho}$ being the area element on $\sigma_{\rho}$.

For $n=1,2, \cdots$ define $S_{k, n}$ to be the set of numbers $\rho$ such that

$$
\int_{\sigma} g_{k}(x) d \sigma_{\rho} \leqq n M
$$

Then from (A1) we see that

$$
\text { meas } S_{k, n} \geqq 1-\frac{1}{n}
$$

Thus

$$
\operatorname{meas}\left(\lim _{k \rightarrow \infty} s_{k, n}\right) \geqq 1-\frac{1}{n} .
$$

Therefore, if $n>1 / \varepsilon$

$$
\left(\limsup _{k \rightarrow \infty} S_{k, n}\right) \cap S \cap(1-\varepsilon, 1)
$$

has positive measure and is thus nonempty, which completes the proof.

To prove (41) take $B_{v, i}=B$. From Rellich's Lemma

$$
D_{k \rightarrow \infty}^{l-1} z_{k}(x) \stackrel{s}{\rightarrow} 0 \text { in } \mathscr{L}^{r}(B)
$$

Using the fact that a convergent sequence in $\mathscr{L}^{1}$ has a subsequence which is convergent a.e.,(A5) implies

$$
D_{h \rightarrow \infty}^{l-1} z_{k_{h}}(x) \stackrel{s}{\rightarrow} 0 \text { in } \mathscr{L}^{r}\left(\sigma_{\rho}\right) \text { for almost every } \rho .
$$

We define $S$ to be the set on which (A6) holds and apply our preliminary result with $g_{h}(x)=\left|D^{l} z_{k_{h}}(x)\right|^{r}$. (41) then follows.

\section{REFERENCES}

1. S. Agmon, A. Douglis and L. Nirenberg, Estimates near the boundary for solutions of elliptic partial differential equations satisfying general boundary conditions. I, Comm. Pure Appl. Math. (4) 12 (1959), 623-727.

2. N. G. Meyers and J. Serrin, $H \equiv W$, Proc. Nat. Acad. Sci. U.S.A. (1) 51 (1964), 1055-1056.

3. C. B. Morrey, Quasi-convexity and the lower semicontinuity of multiple integrals, Pacific J. Math. (1) 2 (1952), 25-53.

4. - Multiple integral problems in the calculus of variations and related topics, Ann. Scuola Norm. Sup. Pisa (1) 14 (1960), 1-61.

5. L. Van Hove, Sur l'extension de la condition de Legendre du calcul des variations aux integrals multiples a plusiers fonctions inconnues, Nederl. Akad. Wetensch. (1) 50 (1947), 18-23.

UNIVERSITY OF MINNESOTA, MinNBAPOLIS, MinNesota 